\title{
Reconstruction of Cranial Defect With Individualized PEEK Implant: Case Report
}

\author{
Cristopher Mayer $\mathrm{O}^{1,2^{*}}(\mathrm{D})$, Tomás Donoso $\mathrm{H}^{3}(\mathrm{D})$, Roberto García $B^{4}(\mathrm{D})$, Claudio Huentequeo $M^{5,6}$ (i) and \\ Francisco Pampin $L^{7}$ (D)
}

${ }^{1}$ Department of Oral and Maxillofacial Surgery, Hospital del Salvador, Santiago, Chile

${ }^{2}$ Department of Oral and Maxillofacial Surgery, Hospital Luis Tisné Brousse, Santiago, Chile

${ }^{3}$ Department of Oral and Maxillofacial Surgery, Hospital Dr. Eduardo Schütz S, P Montt, Chile

${ }^{4}$ Department of Oral and Maxillofacial Surgery, Hospital Clínico Félix Bulnes, Santiago, Chile

${ }^{5}$ Department of Oral and Maxillofacial Surgery, Hospital Dr. Abraham Godoy Peña, Lautaro, Chile

${ }^{6}$ Department of Oral and Maxillofacial Surgery, Universidad de la Frontera, Temuco, Chile

${ }^{7}$ Department of Oral and Maxillofacial Surgery, Universidad de Valparaíso, Chile

*Corresponding author: Cristopher Mayer Olivares, MFS, MSc, Surgeon, Department of Oral and Maxillofacial Surgery, Hospital del Salvador and Hospital Santiago Oriente, Av. Salvador 364, Providencia, Santiago, Chile, Tel: +569-325-200-48

\begin{abstract}
Introduction: Cranioplasty has numerous indications, and its main objective is to support the underlying noble structures, along with restoring shape and aesthetics. The treatment of choice is controversial, and is mainly related to the type of material to be used.
\end{abstract}

Material and methods: We present the case of a 44-yearold woman with a left fronto-temporo-parietaldefect, who underwent a cranioplasty with a customized polyetheretherketone (PEEK) prosthesis.

Results: There were no complications in the perioperative period, nor were there rejection reactions, infection or exposure of the implant in the postoperative period, with satisfactory aesthetic (improvement of the cranial contour) and functional results due to the excellent fit and material properties.

Conclusions: The choice of polyetheretherketone (PEEK) as a reconstructive material in craniofacial defects has proven to be a safe method, with good clinical results when compared to other reconstruction materials.

\section{Keywords}

Cranioplasty, Cranial implants, Polyetheretherketone, Cranial defect, Cranial reconstruction

\begin{abstract}
Abbreviations
PEEK: Polyetheretherketone; HA: Hidroxyapatite, DICOM: Digital imaging and Communications in medicine; CAD: Computer-aided design; CAM: Computer-aided manufacturing; FOV: Field of view
\end{abstract}

\section{Introduction}

The cranioplasty procedure is one of the oldest procedures performed in neurosurgery, and consists of surgically repairing a cranial defect, generally in a delayed manner, in order to decrease the risk of graft infections [1]. There are various indications for this technique, all with the main objective of supporting the underlying noble structures, along with restoring shape and aesthetics [2]. Contraindications include infections, hydrocephalus, and cerebral edema [3].

Cranial defects have various origins: trauma, decompression procedures, tumors, infections, congenital injuries or iatrogenesis [4]. In all these cases, where there is both a morpho-functional and a psychological alteration, cranioplasty has demonstrated its contribution, giving protection to noble structures, restoring aesthet-

Citation: Cristopher MO, Tomás DH, Roberto GB, Claudio HM, Francisco PL (2020) Reconstruction of Cranial Defect With Individualized PEEK Implant: Case Report. Neurosurg Cases Rev 3:040. doi. org/10.23937/2643-4474/1710040

Accepted: August 10, 2020; Published: August 12, 2020

Copyright: (c) 2020 Cristopher MO, et al. This is an open-access article distributed under the terms of the Creative Commons Attribution License, which permits unrestricted use, distribution, and reproduction in any medium, provided the original author and source are credited. 
ics and function, and reducing undesired neurological effects $[4,5]$. There are several ways to reconstruct these defects [6-12], and in all of them it is important to evaluate a series of factors, such as the material to be used, the costs, the type of surgical procedure to be performed, its associated morbidity, and its stability in the time [4].

The current treatment of choice is controversial, and is related to the type of material to be used, since the ideal material for reconstruction of cranial defects does not exist [5], so finding one that improves the prognosis of treatment has been a constant search [3]. With the difficulties associated with the use of autografts, interest in alloplastic materials has grown, and a series of materials that can reduce the complications associated with the use of bone grafts have been analyzed and described, including: Methyl methacrylate, hydroxyapatite (HA), porous polyethylene, ceramics and polyetheretherketone (PEEK), among others $[2,3,13]$.

Polyetheretherketone (PEEK) is a thermoplastic polymeric material with high mechanical properties. PEEK implants have increased in popularity due to 3D design and printing computational technology, which allows implants to be manufactured specifically for each patient [3]. Some of the advantages of using custom implants are the greater anatomical fit they provide (intraoperative adjustment rates close to titanium) and a shorter operative time [4]. The purpose of this article is to share the report of a case, where the reconstruction of a cranial defect was performed using a customized PEEK implant, and to analyze its indications, advantages, and clinical results.

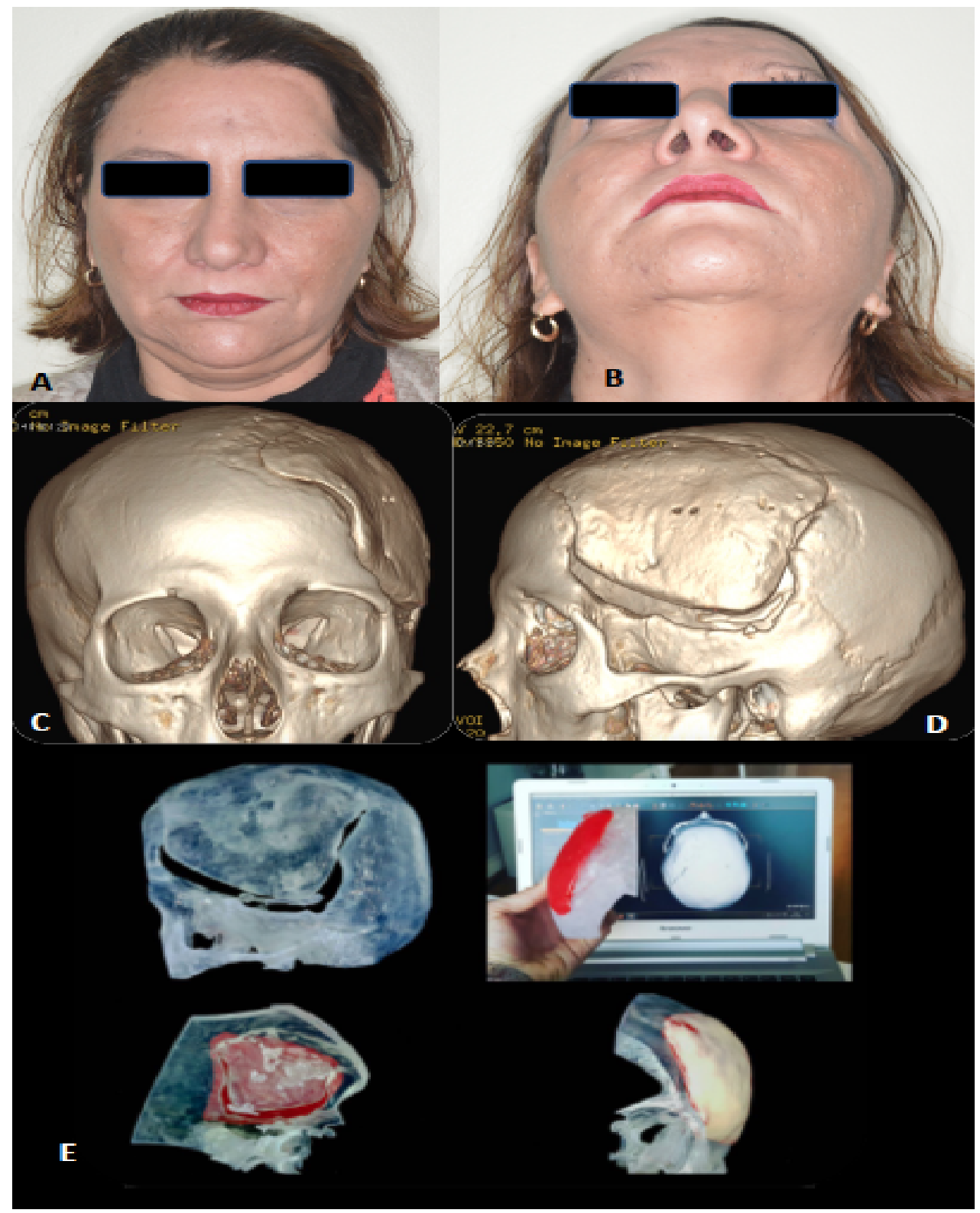

Figure 1: $(A$ and $B)$ Preoperative frontal view of patient with fronto-temporo-parietal left defect. Consecutive to decompressive craniotomy; (C and D) Tridimentional reconstruction of computed tomography (CT) front and lateral view, where previous cranioplasty with polymethylmethacrylate and its mismatch due to material contraction are observed; (E) Impression of a stereolithographic model to evaluate the defect, design and manufacture of a customized PEEK prosthesis. 


\section{Case Report}

44-year-old female patient, with a history of cerebral aneurysm treated by left fronto-temporo-parietal decompressive craniectomy, and a delayed cranioplasty with polymethyl methacrylate (Figure $1 \mathrm{~A}$ and Figure 1B), was referred after five years to the Maxillofacial Surgery Unit of the Hospital del Salvador (Santiago, Chile), for an evaluation and treatment of the residual cranial defect generated by the contraction of polymethyl meth- acrylate, which psychologically affected the patient. On clinical examination, the sinking in the anterior portion of the left temporal fossa is evident, where it is possible to feel the gap or mismatch between the edges of the polymethyl methacrylate prosthesis and the bone tissue. Based on the clinical and imaging history obtained, a removal of the polymethyl methacrylate and the installation of a customized PEEK prosthesis are planned. Pre-operative exams and a craniofacial cone-

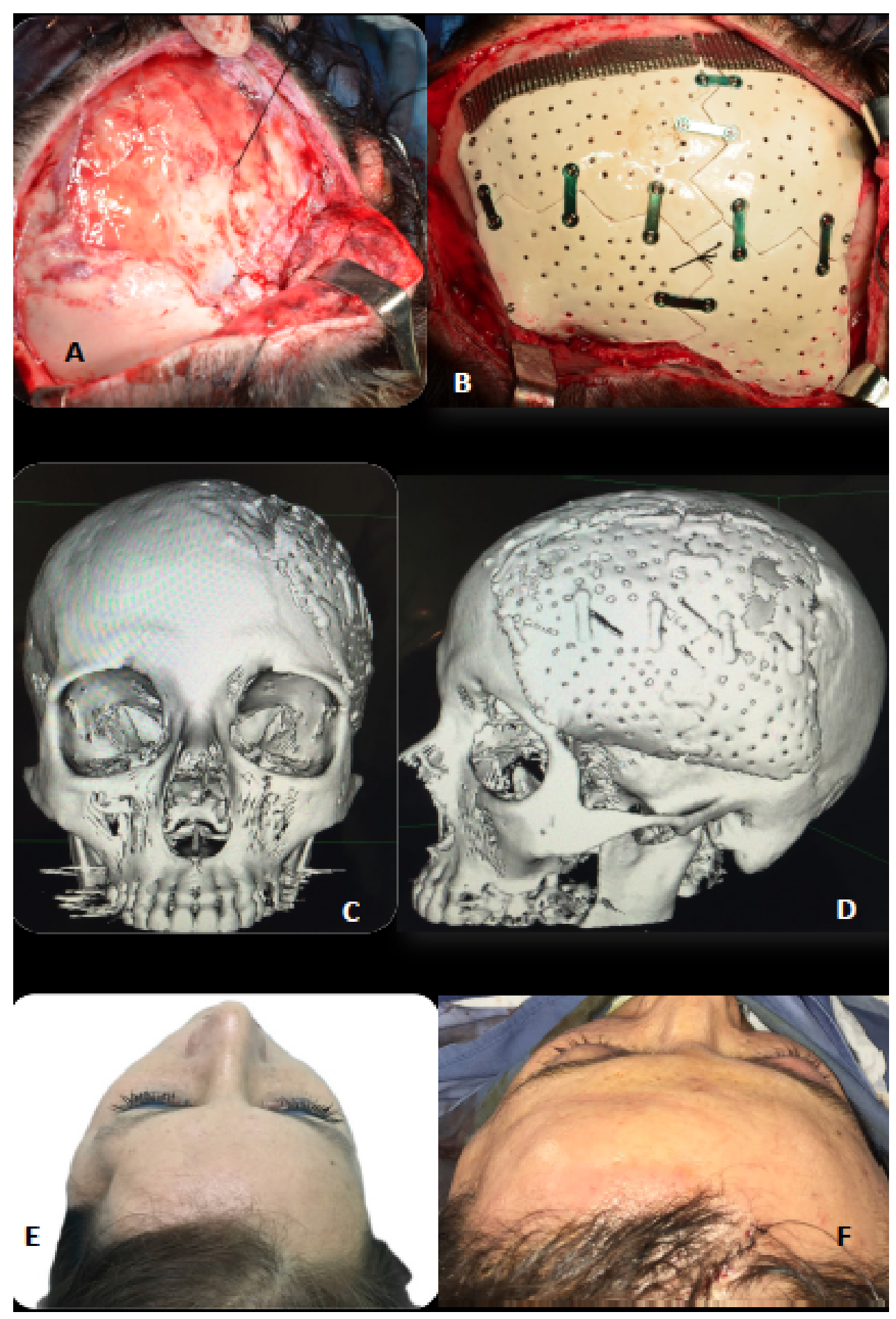

Figure 2: $(A)$ Left hemicoronal approach and cranial defect exposure. Dura mater and suspensory suture are observed; (B) Customized PEEK prosthesis installed and fixed with $1.5 \mathrm{~mm}$ osteosynthesis plates and screws. There is also a reinforcement with titanium mesh; $(C$ and $D)$ Tridimentional reconstruction of control computed tomography (CT) with a customized PEEK prosthesis in the correct position (front and side views); (E) Preoperative cranial defect; (F) Cranial defect in the immediate post-operative period. 
beam computed tomography (CBCT) are requested. All CBCT datasets were acquired using the i-CAT CBCT unit (Classic i-CAT scanner, Imaging Sciences International, Hatfield, PA, USA). The device was operated at $5 \mathrm{~mA}$ and $120 \mathrm{kV}$ using a high frequency, constant potential, with slice thickness of $0.5 \mathrm{~mm}$ and a FOV of $25 \mathrm{~cm}$. The patient was positioned into the device supported by the constructed plastic head holder. The chin was inserted into the chin holder, and vertical and horizontal laser lights on the device used to position the head. Primary reconstruction of the data was performed immediately after acquisition. The DICOM files were then exported to the Mimics software (Materialize, Leuven, Belgium), where the bone tissue was isolated to create a three-dimensional reconstruction of the skull, in order to more fully evaluate the bone defect and the lack of fit of the polymethyl methacrylate (Figure $1 \mathrm{C}$ and Figure 1D). Virtual planning was performed in close collaboration with the company Arcomed (Santiago, Chile), and the manufacturing process was performed entirely by this company. Subsequently, the creation of a stereolithographic model is coordinated with the laboratory, which will be used during the design and manufacture of the customized PEEK prosthesis. The shared evaluation between the laboratory technician and the surgeon allows defining the contours, extension, terminations and screw insertion areas in the prosthesis. Once the design is approved, the file with all the data is sent, and with a rapid prototyping machine, the patient-specific implant is created (Figure 1E). 3D printing files for the designed cranial implant were sent as an STL file to the laboratory where all parts were produced using additive manufacturing or 3D printing technologies, based on a high temperature extrusion system of material filaments. The PEEK implant was manufactured using the $3 \mathrm{~d}$ printer MAGIC-HT-MAX (IEMAI 3D, China) through an extrusion process at an average temperature of $350{ }^{\circ} \mathrm{C}$ with a flow rate of $2.2 \mathrm{mg} / \mathrm{s}$ using filaments with a diameter of $1.75 \mathrm{~mm}$ and with a thickness of construction layer 0.2 $\mathrm{mm}$. Finally, the prosthesis is sent to the hospital to be sterilized before surgery. Under general anesthesia and antibiotic prophylaxis (Cefazolin 2 gr iv), the cranial defect is addressed through the previous hemi coronal approach. In conjunction with the neurosurgeon, the scar tissue was dissected and removed, and adequate hemostasis was performed, completely exposing the defect and the polymethyl methacrylate prosthesis, which was mobile, only held by some wires. Once the prosthesis was removed, the dura was reviewed, finding some perforations which were sutured (Figure 2A and Figure $2 B)$. Due to the size of the prosthesis, it was made in parts, which were assembled and fixed with miniplates and $1.5 \mathrm{~mm}$ screws prior to installation ( ${ }^{\circ}$ Biomateriales Chile). Subsequently, the PEEK implant was positioned in the defect, verifying that its adaptation is adequate. Once the final position was confirmed, suspensory sutures were passed through the preformed holes of the
PEEK prosthesis to fix the dura, and then the implant was fixed and reinforced with $1.5 \mathrm{~mm}$ plates, titanium mesh, and screws ( ${ }^{\circ}$ Biomateriales Chile) in the edges of the defect. Intraoperative adjustments of the prosthesis were not necessary. After hemostasis control, a profuse wash with physiological serum and plane closure of the approach was performed with Vicryl 3-0 and Prolene 4-0. No drainage was left during the postoperative period. Compression dressings were installed at the end of the procedure. A post-operative control tomography was requested (Figure $2 \mathrm{C}$ and Figure 2D), and the patient was hospitalized under observation and pharmacological treatment for 3 days until discharge. There were no complications during the post-operative period (rejection reactions, infection or implant exposure), with a follow-up of 3 years. The final result was aesthetic (improvement of the cranial contour) and functional, due to the excellent fit and material properties (Figure $2 \mathrm{E}$ and Figure 2F).

\section{Discussion}

Cranial defects generate functional, aesthetic and psychological alterations that significantly affect the quality of life of patients [5]. The current controversy in reconstruction focuses on identifying the best time to perform it, and on the type of implant (material) to use $[1,3]$. According to the literature, the ideal material should be radiolucent, resistant to infections, non-conductive to temperature, biomechanically resistant, malleable, with good fit, low cost and capable of promoting tissue growth on it [3].

Currently, autologous bone grafting is considered the "gold standard" for post-craniotomy reconstruction. However, it is not always possible to use it, since in most cases, these reconstructions are performed in a delayed manner (as in the case presented), compromising the vitality of the graft [5]. Regarding the size of the defect to be reconstructed, the Poukens classification describes small defects $\left(10-50 \mathrm{~cm}^{2}\right)$ which can be reconstructed with autologous bone graft segments or non-personalized titanium meshes. For major defects $\left(50-100 \mathrm{~cm}^{2}\right)$, the use of preserved autologous bone (by cryopreservation or in abdominal pockets) of calvarial bone, or allogenic materials [14] is described.

However, this is related to a series of problems, such as the biological and morphological changes experienced by the graft due to loss of vitality, which translates into unpredictable reabsorptions, and only osteoconductive properties [2,3]. Other authors establish that for defects greater than $25 \mathrm{~cm}^{2}$, custom implants are indicated [15]. The cranial defect of the case presented in this article had a size greater than $25 \mathrm{~cm}^{2}$, for which reason we decided to manufacture a custom prosthesis to reconstruct the defect.

Customized and non-customized titanium implants have been widely used, however, despite their great 
properties such as biocompatibility or the possibility of osseointegration, they have a number of disadvantages, such as structural resistance ten times greater than that of cortical bone (1000 MPa against $100 \mathrm{MPa}$ ), and temperature conduction, which generates a series of harmful effects on the brain [6]. On the other hand, PEEK individualized implants are presented as an excellent alternative, fulfilling the requirement of structural resistance, and adequate rigidity and elasticity, which reduces the stress transmitted to noble structures such as the brain $[2,16]$.

PEEK, is a semi-crystalline, thermoplastic, hydrophobic, and chemically inert polymer, which has demonstrated advantages such as: Radiolucency, not generating artifacts in imaging studies, biomechanical properties (resistance, thickness and elasticity) comparable to cortical bone, does not conduct temperature, it can be sterilized, it can wear out, it has a good fit, and it is lighter than other materials $[2,3,13]$. Among its disadvantages are described: The high cost, risk of infections, and the absence of osseointegrative properties, which is why the long-term stability of these implants is given by their three-dimensional design and fixation with osteosynthesis elements $[6,13]$. This is why, in the case of the patient, in addition to the fixation with an osteosynthesis element, an area was reinforced with titanium mesh.

The first series of cases of delayed cranial reconstruction with PEEK prostheses was published in 2006 with promising results [12]. Virtual planning is essential for the use of this type of prosthesis, which reduces intraoperative times and complications [5]. Precast implants can be obtained either directly or indirectly. The indirect process is performed using a template obtained from a stereolithography, and direct manufacturing is a virtual process that relies on computer-aided design and manufacturing (CAD-CAM) to produce a custom implant [17]. The tomographic examination must be without contrast, with cuts no greater than $0.5 \mathrm{~mm}$, and with a field of vision or FOV that allows the entire skull to be covered. It is recommended that this examination not be older than six months, due to bone resorption that occurs over time at the edges of the defect [6].

There are retrospective studies that show a good acceptance by patients of PEEK prostheses, with adequate aesthetics and functionality $[1,18]$. However, there are certain considerations when using it, such as avoiding its use when the defect is in direct relation to the sinuses (due to the risk of infection), and disinfecting and sterilizing the implant before installation $[5,6]$.

Possible predictors or risk factors for complications have also been described, including patients with associated vascular comorbidity, smokers, very late reconstructions, prolonged surgical times, and patients with local infections and previous surgeries [1,2]. Among the complications in relation to the customized PEEK prostheses, different studies report infections, post-operative hematomas, fistulas, seromas, dehiscence and problems in the healing of operative wounds [1-5]. The type of material used would not be a risk factor associated with infections, however, it has been seen that the materials with the largest surface have greater resistance to infections, since they allow active support and growth of the tissues towards the interior. Therefore, the manufacturing methods and the physical structure of these implants can positively influence by reducing the risk of infections $[2,6,7]$.

\section{Conclusions}

The choice of PEEK as a reconstructive material in craniofacial defects has proven to be a safe method. Cranioplasty with custom PEEK implants continue to give satisfactory results when compared to other types of cranial reconstruction. Furthermore, these implants have the advantage of being sterilizable, giving the option of being reused. The high costs of this type of treatment are justified when surgical and hospitalization times are shortened, which improves the prognosis of treatment.

\section{Conflict of Interest}

The authors have no commercial or financial associations that might create a conflict of interest with the information presented in this manuscript.

\section{Statement of Equal Authors Contribution}

All authors have contributed equally to the work.

\section{References}

1. Jonkergouw J, van de Vijfeijken SECM, Nout E, Theys T, Van de Casteele E, et al. (2016) Outcome in patient-specific PEEK cranioplasty: A two-center cohort study of 40 implants. J Cranio-Maxillofacial Surg 44: 1266-1272.

2. Kwarcinski J, Boughton P, Ruys A, Doolan A, van Gelder J (2017) Cranioplasty and craniofacial reconstruction: A review of implant material, manufacturing method and infection risk. Appl Sci 7: 1-17.

3. Shah AM, Jung H, Skirboll S (2014) Materials used in cranioplasty: A history and analysis. Neurosurg Focus 36: 1-7.

4. Gonzalez de Santiago MA, Chaurand Lara J, Sandoval B (2011) Reconstruccion craneal con implante personalizado. Rev Esp Cir Oral y Maxilofac 33: 40-44.

5. Alonso-Rodriguez E, Cebrian-Carretero JL, del Castillo JL, Hernandez-Godoy J, Nieto MJ, et al. (2016) Complicaciones en reconstruccion craneal con protesis de polieteretercetona. Rev Esp Cir Oral y Maxilofac 38: 199-205.

6. Lethaus B, Poort Ter Laak M, Laeven P, Beerens M, Koper D, et al. (2011) A treatment algorithm for patients with large skull bone defects and first results. J Cranio-Maxillofacial Surg 39: 435-440.

7. Blake GB, MacFarlane MR, Hinton JW (1990) Titanium in reconstructive surgery of the skull and face. Br J Plast Surg 43: 528535.

8. Park HK, Dujovny M, Agner C, Diaz FG (2001) Biomechanical properties of calvarium prosthesis. Neurol Res 23: 267-276.

9. Eufinger $\mathrm{H}$, Wehmoller M (1998) Individual prefabricated titani- 
um implants in reconstructive craniofacial surgery: Clinical and technical aspects of the first 22 cases. Plast Reconstr Surg 102: 300-308.

10. Cho-Lee GY, Naval-Gias L, Gonzalez-Garcia R, Martos-Diaz PL, Mario Fernando Muñoz-Guerra, et al. (2010) Bifocal transport osteogenesis for the reconstruction of adult calvarial defects: A new surgical technique. J Cranio-Maxillofacial Surg 38: 368-373.

11. Hattar S, Asselin A, Greenspan D, Oboeuf M, Berdal A, et al. (2005) Potential of biomimetic surfaces to promote in vitro osteoblast-like cell differentiation. Biomaterials 26: 839-848.

12. Hanasono MM, Goel N, DeMonte F (2009) Calvarial reconstruction with polyetheretherketone implants. Ann Plast Surg 62: 653-655.

13. Zubillaga Rodriguez I, Sanchez Aniceto G, Montalvo Moreno JJ (2007) Reconstruccion craneal con biomateriales. Revision historica y estado actual. Revista Espanola de Cirugia Oral y Maxilofacial 29: 79-89.
14. Poukens J, Laeven P, Beerens M, Nijenhuis G, Vander Sloten J, et al. (2008) A classification of cranial implants based on the degree of difficulty in computer design and manufacture. Int J Med Robot Comput Assist Surg 4: 46-50.

15. Kuttenberger JJ, Hardt N (2001) Long-term results following reconstruction of craniofacial defects with titanium micro-mesh systems. J Cranio-Maxillofacial Surg 29: 75-81.

16. Lethaus B, Safi Y, Ter Laak-Poort M, Kloss-Brandstatter A, Banki F, et al. (2012) Cranioplasty with customized titanium and PEEK implants in a mechanical stress model. J Neurotrauma 29: 10771083.

17. Eufinger $\mathrm{H}$, Wehmoller $\mathrm{M}$ (2002) Microsurgical tissue transfer and individual computer-aided designed and manufactured prefabricated titanium implants for complex craniofacial reconstruction. Scand J Plast Reconstr Surg Hand Surg 36: 326-331.

18. Rosenthal G, Ng I, Moscovici S, Lee KK, Lay T, et al. (2014) Polyetheretherketone implants for therepair of large cranial defects: A 3-center experience. Neurosurgery 75: 523-529. 